\title{
Characterization of a potential ABC-type bacteriocin exporter protein from Treponema denticola
}

\author{
Kimiko Tanaka-Kumazawa', Yuichiro Kikuchi²,3, Yumiko Sano-Kokubun', Seikou Shintani', Masashi Yakushiji', \\ Howard K. Kuramitsu ${ }^{4}$ and Kazuyuki Ishihara $2,3^{*}$
}

\begin{abstract}
Background: Treponema denticola is strongly associated with the development of periodontal disease. Both synergistic and antagonistic effects are observed among bacterial species in the process of biofilm formation. Bacteriocin-related genes have not yet been fully characterized in periodontopathic bacteria. The aim of this study was to detect and characterize bacteriocin-associated proteins in T. denticola.

Methods: The whole genome sequence of T. denticola ATCC 35405 was screened with a Streptococcus mutans bacteriocin immunity protein (ImmA/Bip) sequence. The prevalence of homologous genes in $T$. denticola strains was then investigated by Southern blotting. Expression of the genes was evaluated by qRT-PCR.

Results: In the genome sequence of $T$. denticola, an amino acid sequence coded by the open reading frame TDE_0719 showed $26 \%$ identity with the S. mutans ImmA. Furthermore, two protein sequences encoded by TDE_0425 and TDE_2431 in T. denticola ATCC 35405 showed $40 \%$ identity with that coded by TDE_0719. Therefore, TDE_0425, TDE_0719, and TDE_2431 were designated as tepA1, A2, and A3, respectively. Open reading frames showing similarity to the $\mathrm{Hly} \mathrm{D}$ family of secretion proteins were detected downstream of tepA1, $A 2$, and $A 3$. They were designated as tepB1, B2, and B3, respectively. A gene harboring a bacteriocin-like signal sequence was detected upstream of tepA1. The prevalence of tepA1 and $A 2$ differed among Treponema species. Susceptibility to chloramphenicol and ofloxacin was slightly decreased in a tepA2 mutant while that to kanamycin was increased. Expression of tepA3-B3 was increased in the tepA2 mutant.

Conclusion: These results indicate that T. denticola ATCC 35405 has three potential bacteriocin export proteins and that the presence of these genes differs among the Treponema strains. TepA3-B3 of the corresponding proteins may be involved in resistance to chloramphenicol.
\end{abstract}

Keywords: ABC transporter, Bacteriocin, Antimicrobial agent susceptibility, Treponema denticola

\section{Background}

Treponema denticola is a spiral-shaped motile rod that is frequently isolated from the periodontal pockets of subjects with chronic periodontitis $[1,2]$ and that possesses several potential virulence factors [3]. It is often co-isolated with Porphyromnas gingivalis and Tannerella forsythia from the dental plaque biofilms of chronic

\footnotetext{
*Correspondence: ishihara@tdc.ac.jp

2Department of Microbiology, Tokyo Dental College, 2-9-18 Misaki-cho,

Chiyoda-ku, Tokyo 101-0061, Japan

${ }^{3}$ Oral Health Science Center, Tokyo Dental College, 2-9-18 Misaki-cho,

Chiyoda-ku, Tokyo 101-0061, Japan

Full list of author information is available at the end of the article
}

periodontitis patients [4] and is involved in the development of periodontitis $[5,6]$.

Interactions among bacterial species that reside in dental plaque biofilms influence the composition of the biofilms [7-9]. Microorganisms in biofilms develop their niche by symbiosis with other microorganisms and suppression of competitors by secreting antagonistic factors $[10,11]$. To suppress the growth of a competitor, various microorganisms produce antimicrobials such as bacteriocins and $\mathrm{H}_{2} \mathrm{O}_{2}$ [12]. These antimicrobial factors play an important role in the survival strategy of the microorganisms in the biofilms. Similarly, in dental plaque 
biofilms, many species of microorganisms have been reported to produce bacteriocins or bacteriocin-like substances [13-18]. In the bacteriocin-producing bacteria, genes encoding a bacteriocin, a bacteriocin ABC transporter, and bacteriocin immunity proteins are involved in bacteriocin production [19]. Bacteriocin immunity proteins protect the microbes against the effects of their own bacteriocins [20]. Bacteriocin ABC transporters have two functional domains: a peptidase C39 domain, which is involved in the processing of bacteriocin precursors at the double glycine, and an $\mathrm{ABC}$ transporter domain, which is involved in the export of the bacteriocins [21]. A synergistic effect between T. denticola and $P$. gingivalis has been reported [22], while growth of $T$. denticola was inhibited by plaque-associated Streptococcus mutans [23]. The antagonistic effects produced by bacteriocins have been genetically characterized in oral streptococci [24-26], while those in periodontopathic bacteria remain to be established. Suppressing the growth of and avoiding inhibition by competitors would benefit $T$. denticola in the colonization of the subgingival plaque and the development of periodontopathic biofilms.

In the present study, we intended to characterize bacteriocin-associated proteins from $T$. denticola. By screening of $T$. denticola genomic DNA using $S$. mutans bacteriocin immunity protein, ABC-type bacteriocin exporter-like proteins were detected and the function of the exporter was investigated.

\section{Methods}

\section{Bacterial strains and culture conditions}

The strains of $T$. denticola used in this study are listed in Table 1. The strains were maintained in TYGVS medium [27] at $37{ }^{\circ} \mathrm{C}$ under anaerobic conditions (10\% $\mathrm{CO}_{2}, 10 \% \mathrm{H}_{2}$, and $80 \% \mathrm{~N}_{2}$ ) in an anaerobic chamber (Hirasawa, Tokyo, Japan). For the mutant strain, TYGVS containing $40 \mu \mathrm{g} / \mathrm{ml}$ erythromycin was used.

\section{Sequence homology-based screening}

The whole genome sequence of $T$. denticola ATCC 35405 in the Los Alamos oral pathogen database (http://www.oralgen.org) was screened for homologous

Table 1 List of the strains used in this study

\begin{tabular}{|c|c|c|}
\hline Bacterial strain & Relevant characteristics & Source or reference \\
\hline T. denticola ATCC 33520 & $\mathrm{Em}^{\mathrm{s}}$ & {$[41]$} \\
\hline T. denticola ATCC 33521 & $\mathrm{Em}^{\mathrm{s}}$ & {$[41]$} \\
\hline T. denticola ATCC 35404 & $\mathrm{Em}^{\mathrm{s}}$ & [41] \\
\hline T. denticola ATCC 35405 & $\mathrm{Em}^{\mathrm{s}}$ & {$[41]$} \\
\hline T. denticola GM1 & $\mathrm{Em}^{\mathrm{s}}$ & {$[42]$} \\
\hline T. denticola KT-3 & tepA2:: $\mathrm{Em}^{\mathrm{r}}$ & This study \\
\hline
\end{tabular}

sequences with the $S$. mutans bacteriocin immunity protein (ImmA/Bip) sequence [28] using the protein blast program. The obtained homologous sequences were further compared against the database of National Center for Biotechnology Information (NCBI, http://blast.ncbi.nlm.nih.gov/Blast.cgi). The DNA sequences coding for the homologous proteins in $T$. denticola were designated as tepA1, $A 2$, and $A 3$ as described in the Results section, and they were characterized with Genetyx-MAC v. 17.0.6 (Genetyx Corporation, Tokyo, Japan).

\section{Southern blotting}

The prevalence of DNA sequences homologous to tepA1, $A 2$, and $A 3$ in $T$. denticola species was detected by Southern blotting. T. denticola was grown for 3 days in TYGVS medium and genomic DNA was isolated with the Gentra Puregene Cell Kit (Qiagen, Tokyo, Japan). Southern blot analysis was performed as described previously [29]. Digoxigenin-labeled primer was synthesized on a GeneAmp 9700 thermal cycler (Life Technologies, Carlsbad, CA) with the PCR DIG Probe Synthesis Kit (Roche Diagnostics, Tokyo, Japan) according to the manufacturer's instructions using the 3 primer pairs I-1F and $\mathrm{I}-1 \mathrm{R}, \mathrm{I}-2 \mathrm{~F}$ and $\mathrm{I}-2 \mathrm{R}$, and $\mathrm{I}-3 \mathrm{~F}$ and $\mathrm{I}-3 \mathrm{R}$, for tep $A 1$, tep $A 2$, and tepA3, respectively (Table 2). Hybridized bands were detected with the DIG Nucleic Acid Detection Kit (Roche Diagnostics).

\section{Construction of a tepA2 mutant}

As TepA2 showed similarity to ImmA, a tepA2-deficient mutant of T. denticola ATCC 35405 was constructed by allelic exchange mutation to investigate the role of tepA2. Briefly, two fragments flanking the tepA2 gene were amplified with primer pairs 718D/719U and 719D/ $720 \mathrm{U}$ (listed in Table 2), respectively. The ermF-ermAM cassette was amplified with the primers EMD2 and EMU2 and the fragment was inserted between the upstream and downstream fragments using the PCR-based overlap-extension method [30]. The constructed fragments were introduced by electroporation and transformants were isolated on TYGVS agar plates containing $40 \mu \mathrm{g} / \mathrm{ml}$ erythromycin as described previously [29]. Inactivation of the gene in mutant KT-3 was confirmed by Southern blot and PCR analyses.

\section{Antibiotic susceptibility testing}

The effect of tepA2 inactivation on the susceptibility of $T$. denticola to antibiotics was investigated. Chloramphenicol, ofloxacin, and kanamycin, to which $T$. denticola showed low susceptibility in our preliminary results, were selected. T. denticola ATCC 35405 and KT-3 were cultured as described above for 4 days. The cells were adjusted to an optical density at $660 \mathrm{~nm}\left(\mathrm{OD}_{660}\right)$ of 0.1 with TYGVS medium using a spectrophotometer (UV- 
Table 2 List of gene-specific primers used in this study

\begin{tabular}{|c|c|}
\hline $\begin{array}{l}\text { Primers and } \\
\text { probes }\end{array}$ & Sequence \\
\hline $\mathrm{I-1F}$ & 5'-AAATTTGCAAAGGCCTACCGTGAGCTT-3' \\
\hline I-1R & 5'-TTTCGACAAAAGAGTGTACTCCCGTTTCC-3' \\
\hline $\mathrm{I}-2 \mathrm{~F}$ & 5'-CGGGCGGTATACTCATGCTGATTGCC-3' \\
\hline $\mathrm{I}-2 \mathrm{R}$ & 5'-TTTGCCTGAACCGGCTCCTAC-3' \\
\hline $\mathrm{I}-3 \mathrm{~F}$ & 5'-CGGAGAACTTGTTGCAAGGATGAACGATAC-3' \\
\hline I-3R & 5'-TCCCGAAAAACAAGAGAATGTCCTGAGGAAC-3' \\
\hline $718 \mathrm{D} 2$ & 5'-GGTTTGCTCTTGCAATTCCCATATTTA-3' \\
\hline $719 U$ & $\begin{array}{l}\text { 5'-TGTTGCAAATACCGATGAGCAAAATAATATGAGAACGCA } \\
\text { CCGCAGAA-3' }\end{array}$ \\
\hline EMD2 & 5'-GCTCATCGGTATTTGCAACATCATAG-3' \\
\hline EMU2 & 5'-CTACATTCCCTITAGTAACGTGTAACTTTC-3' \\
\hline 7190 & $\begin{array}{l}\text { 5'-CGTTACTAAAGGGAATGTAGCTATTTTGACGGGTTGGAG } \\
\text { TTCCAACA-3' }\end{array}$ \\
\hline $720 U$ & 5'-TACGGTACCTGAATAAGCAGCCTTACC-3' \\
\hline tepA1F & 5'-TGCCGTGCAAATGACTCTCT-3' \\
\hline tepA1R & 5'-TTTAAAAACTGCCTACCCAATAAACGC-3' \\
\hline tepA1Pa & 5'-CACAGCTTGGAACTTT-3' \\
\hline tepB1F & 5'-TGAAAAAATTATGGCTTGAAGCACTTGA-3' \\
\hline tepB1R & 5'-TGCCATATCTGCCTTGTATTTTAACTCT-3' \\
\hline tepB1Pa & 5'-CCTGCAACAGCAATTC-3' \\
\hline tepA3F & 5'-CACTCCTGTATTGTTGAAAGTCTTAACG-3' \\
\hline tepA3R & 5'-CACTTACGATTITAAACTCGGCTCTT-3' \\
\hline tepA3P $\mathrm{P}^{\mathrm{a}}$ & 5'-TTGGGTGCCGAATCTA-3' \\
\hline tepB3F & 5'-AGAAAGTTTAAAACTITTTACAGTCTATGCTCCT-3' \\
\hline tepB3R & 5'-CATTATCCCCGCAGTTAAGAGATGA-3' \\
\hline tepB3P $\mathrm{P}^{\mathrm{a}}$ & 5'-TTCCTGCACTTCTCCC-3' \\
\hline tetRF & 5'-CGCAACGCCGGTTCTTAAAA-3' \\
\hline tetRR & 5'-CCTTCGAACAACAGACAATCAGTTT-3' \\
\hline tetRP ${ }^{a}$ & 5'-TCGCATCCCAATTATC-3' \\
\hline $16 S F$ & 5'-GCCGATGATTGACGCTGATATAC-3' \\
\hline $16 S R$ & 5'-CGGACTACCAGGGTATCTAATCCT-3' \\
\hline $16 \mathrm{SP}$ & 5'-CTCCCCGCACCTTC-3' \\
\hline
\end{tabular}

Boldface sequences overlap with the $5^{\prime}$ or $3^{\prime}$ end of ermF-ermAM, aTaqman probe

2550, Shimadzu, Kyoto, Japan), and $100 \mu$ of the cell suspension was added to TYGVS containing $0.5-1 \mu \mathrm{g} /$ $\mathrm{ml}$ of chloramphenicol, $16-64 \mu \mathrm{g} / \mathrm{ml}$ of kanamycin, or $8-32 \mu \mathrm{g} / \mathrm{ml}$ of ofloxacin. After incubation for 7 days under anaerobic conditions, cell growth was measured at $\mathrm{OD}_{660}$ with the spectrophotometer.

\section{DNA microarray analysis}

T. denticola ATCC 35405 and KT-3 were cultured as described above for 2 days. To investigate the relation between inactivation of tepA2 and increase of chloramphenicol resistance, exponentially growing cells $\left(\mathrm{OD}_{660}\right.$ $\sim 0.2)$ were incubated with chloramphenicol $(1 \mu \mathrm{g} / \mathrm{ml})$ for $4 \mathrm{~h}$. The cells were harvested immediately after chloramphenicol treatment and total RNA was extracted using Trizol (Life Technologies). DNase treatment was carried out using a TURBO DNA-free kit Life Technologies). cDNA was synthesized using a SuperScript Double-Stranded cDNA Synthesis kit (Invitrogen). DNA microarray gene expression analysis was carried out using Roche NimbleGen custom arrays (2006-0727_TI243275_60mer; Roche, Indianapolis, IN) according to the standard NimbleGen procedure (NimbleGen arrays user's guide: gene expression analysis, v6.0). Briefly, cDNA $(0.5-1 \mu \mathrm{g})$ was labeled using a NimbleGen one-color labeling kit, in which Cy3 was randomly incorporated into the newly synthesized DNA by the Klenow fragment. Labeled cDNA (3 $\mu \mathrm{g})$ derived from each RNA sample was hybridized with each array for 16 to $18 \mathrm{~h}$. The slides were washed, spun dry, and scanned with an Agilent Microarray Scanner with a resolution of $5 \mu \mathrm{m}$. Normalization was carried out with the NimbleScan 2.6.0.0 built-in normalization function. Target genes were confirmed by real-time PCR analysis using primers listed in Table 2.

\section{Quantitative reverse transcription (qRT) PCR expression analysis of tepA1-B1, tepA3-B3, and TDE_0820}

To investigate the relationships among tep $A 1, A 2$ and $A 3$, expression of tepA1 and $\mathrm{A} 3$, tepB1 and $B 3$, and TDE_0820 in the wild-type strain and KT-3 were evaluated. T. denticola ATCC 35405 and KT-3 were cultured as described above. Cells at mid-log phase $\left(\mathrm{OD}_{660}\right.$ of 0.4-0.6) were harvested and total RNA was extracted using Trizol (Life Technologies). DNase treatment was carried out using a TURBO DNA-free kit (Life Technologies) and cDNA was synthesized using ReverTra Ace (Toyobo, Osaka, Japan). Gene expression was measured with real-time PCR using primers and the Taqman probe (Life Technologies) listed in Table 2 on a 7500 Real-Time PCR System (Life Technologies). Expression of each gene was normalized to the level of $16 \mathrm{~S}$ rRNA as an internal control and was expressed as a fold modulation relative to the wild-type strain grown without chloramphenicol.

\section{Statistical analysis}

Comparisons of gene expression and susceptibility to antibiotics were carried out using Student's $t$-test. One-way ANOVA followed by Tukey's multiple comparison test was used for comparisons of gene expression among the two strains grown with or without chloramphenicol. All tests were carried out using Prism v. 5f (GraphPad software, San Diego, CA). The level of significance for all statistical tests was set at $P<0.05$. 


\section{Results}

\section{Screening of homologous sequences for bacterial} immunity proteins in $T$. denticola

A search against the $T$. denticola ATCC 35405 whole genome sequence for sequences homologous to $S$. mutans ImmA revealed an amino acid sequence that possesses $26 \%$ identity with ImmA between residues 5 and 70 (Fig. 1). The sequence is coded by a 2154-bp open reading frame, TDE_0719, and consists of 717 amino acids. The calculated molecular mass of the deduced amino acid sequence is 80279.25 , and the estimated pI is 8.76. The TDE_0719 sequence was compared to the NCBI nucleotide sequence database. The DNA sequence from bp 1408 to 2059, which codes for amino acid residues 469 to 686 , shows $60-70 \%$ identity with ABC transporters of $T$. denticola ATCC 35405, Clostridium botulinum, and Spirochaeta caldaria. This region encodes part of a potential ATP-binding site. The amino acid sequence shows $45 \%$ identity with the bacteriocin ABC transporter of Spirochaeta africana DSM 8902 in a 713-amino-acid overlap, $46 \%$ identity with that of Clostridium lentocellum DSM 5427 in a 713amino-acid overlap, and $47 \%$ identity with that of Clostridium clariflavum DSM 19732 in a 710-amino-acid overlap. In addition to these sequences, amino acid sequences deduced from TDE_0425 and TDE2431 in $T$. denticola ATCC 35405, which were not detected in the first screening using $S$. mutans ImmA, also show homology with that coded by TDE_0719 (43 \% in a 715amino-acid overlap and $40 \%$ in a 710-amino-acid overlap, respectively).

A search of the NCBI Conserved Domain Database (http://www.ncbi.nlm.nih.gov/Structure/cdd/cdd.shtml) revealed that amino acid residues 1-140 of TDE_0719 display similarity to the peptidase $\mathrm{C} 39 \mathrm{~B}$ domain, residues 160-420 to the ABC membrane superfamily, and residues 480-717 to the P-loop NTPase superfamily. Domains of the $\mathrm{ABC}$ membrane superfamily and the P-loop NTPase superfamily are common features of bacterial transporters [31]. Multiple comparisons with other bacteriocin $\mathrm{ABC}$ transporters are depicted in Fig. 2. The Q, $\mathrm{C}$, and $\mathrm{H}$ residues form the putative active site of a subfamily of the peptidase family C39, which mostly consists of bacteriocin-processing endopeptidases from bacteria
[32], and are conserved in the corresponding regions of TDE_0425, TDE_0719, and TDE2431. The ABC transporter signature motif LSGGQRQRIA and GS-KTT, Q, $\mathrm{DE}$, and $\mathrm{H}$, which are observed at ATP-binding sites [33], were highly conserved in the corresponding regions of the $T$. denticola sequences. We designated the three open reading frames TDE_0425, TDE_0719, and TDE2431 as Treponema exporter proteins A1, A2, and A3 (tepA1, tep $A 2$, and tep $A 3$ genes), respectively.

TDE_0720, which is located immediately downstream of TDE_0719, displayed similarity to the multidrug resistance efflux pump of Spirochaeta africana DSM 8902 (22\% identity in a 369-amino-acid overlap) and the HlyD family secretion protein of Desulfosporosinus sp. OT (27 \% identity in a 459 amino-acid overlap). The region of TDE_0720 spanning residues 240 to 350 had similarity to a domain of a HlyD family secretion protein (pfam13437) that is reported to be part of an accessory protein for $\mathrm{ABC}$ exporters of gram-negative bacteria for translocating proteins across the outer membrane [31]. Downstream of tepA1 and tepA3, TDE_0426 and TDE2430 were detected, and both also showed similarity to sequences coding HlyD family secretion proteins. The presence of the C39B peptidase domain, a conserved ATP-binding motif, a membrane-spanning domain, and a nearby accessory protein facilitating export is consistent with the properties of a bacterial $A B C$ exporter. Therefore, we designated TDE_0426, TDE_0720, and TDE2430 as Treponema exporter proteins B1, B2, and $\mathrm{B} 3$ (tepB1, tepB2, and tepB3), respectively.

Bacteriocin transporters are reported to cleave the double-glycine leader peptides from the precursors of bacteriocins for their secretion [31]. A search of the flanking regions of tepA1, $A 2$, and $A 3$ in the genome sequence of $T$. denticola ATCC 35405 revealed that proteins coded by three open reading frames (TDE_0416, TDE_0422, and TDE_0423) upstream of tepA1 have double-glycine bacteriocin-type signal domains. However, no double glycine-containing protein-coding sequence exists near tep $A 2$ and $A 3$. Of the three proteins, those coded by TDE_0422 and TDE_0424 showed high overall similarity (92 \%). The protein coded by TDE_0416 showed high sequence identity with TDE_0422 and TDE_0424 (98\% and 84\%, respectively);

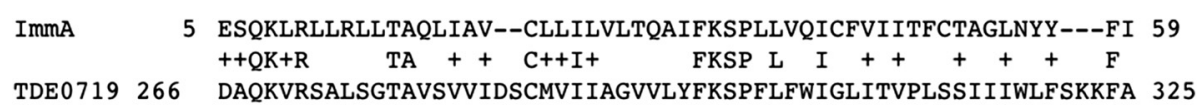

Fig. 1 Homology between the S. mutans bacteriocin immunity protein ImmA and the deduced amino acid sequence of TDE_0719 in T. denticola ATCC35405 


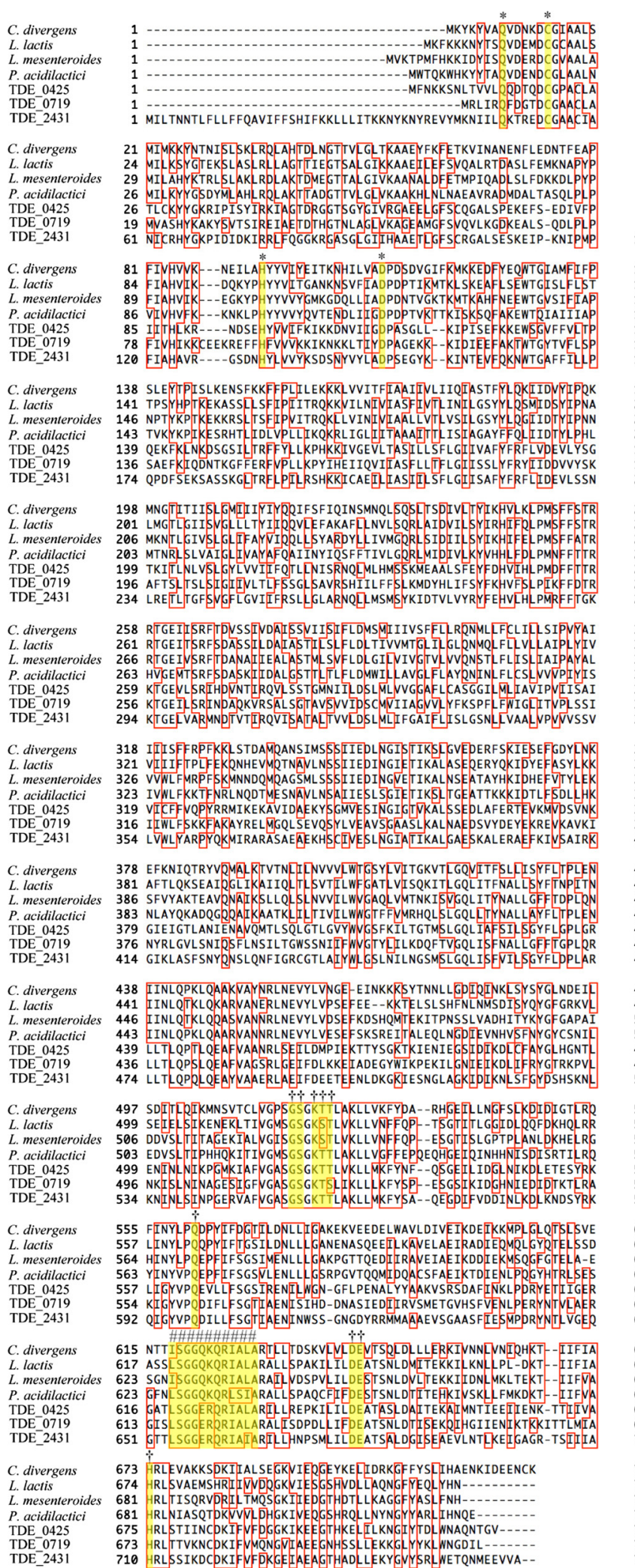

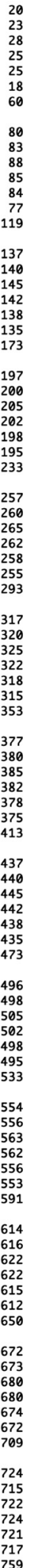

Fig. 2 (See legend on next page.) 
(See figure on previous page.)

Fig. 2 Multiple sequence alignments of bacteriocin $A B C$ transporters. Cysteine and histidine, which are part of the putative active site of the peptidase family C39B, as well as glutamine, which contributes to the oxyanion pore in other cysteine protease families, are marked with * and indicated in yellow. The ATP-binding site and ABC transporter signature motifs are indicated in yellow and marked with + and \#, respectively. The alignment was carried out using the program Genetyx-Mac 16.0.9. C. divergens: ATP-dependent transporter of Carnobacterium divergens, L. lactis: Lactococcin-A transport/processing ATP-binding protein LcnC of Lactococcus lactis subsp. lactis, L. mesenteroides: Mesentericin-Y105 transport/processing ATP-binding protein MesD of Leuconostoc mesenteroides, P. acidilactici: Pediocin PA-1 transport/processing ATP-binding protein PedD of Pediococcus acidilactici, TDE_0425: tepA1, TDE_0719: tepA2, TDE_2431: tepA3

however, the sequence was truncated at residue 167 . TDE_0422 and TDE_0424 showed only weak similarity to penicillin-binding proteins of Bacillus cereus VD148 (37\% identity in a 89-amino-acid overlap).

\section{Prevalence of tepA1, $A 2$, and $A 3$ in $T$. denticola strains}

We examined the presence of the three tepA1, $A 2$, and $A 3$ sequences in $T$. denticola strains ATCC 33520, ATCC 33521, ATCC 35404, ATCC 35405, and GM1 by Southern blotting (Fig. 3). As a single band of approximately $9 \mathrm{kbp}$ was detected in ATCC33520, ATCC33521, and ATCC 35405 with the tep $A 1$ probe; a band at approximately $2 \mathrm{~kb}$ was detected from ATCC 35405 and GM1 with the tepA2 probe; and all strains exhibited two bands at approximately $1.5 \mathrm{~kb}$ and $0.8 \mathrm{~kb}$ with the tep $A 3$ probe.

\section{Antimicrobial sensitivity of the tepA2-deficient mutant}

Among the three bacteriocin transporter-like sequences, we focused on TepA2 because it showed the highest similarity to ImmA. Bacteriocin-like sequences were not detected around tepA2 and $B 2$. Therefore, it is possible that the protein transports other molecules in addition to a bacteriocin. To characterize the function of tepA2 and the functional interaction among the three exporter genes, a tepA2-deficient mutant of strain ATCC 35405 was constructed and designated KT-3. Inactivation of tepA2 did not affect the growth of $T$. denticola (data not shown). Various ABC export systems transport non-protein molecules such as lipophilic drugs, antibiotics, and polysaccharides [31]. To investigate the possible involvement of tepA2 in antibiotic export, the susceptibility of strain 35405 against chloramphenicol, kanamycin, and ofloxacin was evaluated. In the presence of $1 \mu \mathrm{g} / \mathrm{ml}$ chloramphenicol, the growth of the wild type was completely inhibited while $T$. denticola KT-3 showed $40.0 \%$ growth relative to the control (Fig. 4). In the presence of $16-64 \mu \mathrm{g} / \mathrm{ml}$ of kanamycin, the growth of $T$. denticola KT-3 was significantly lower than that of the wild-type strain. In the presence of $16-32 \mu \mathrm{g} / \mathrm{ml}$ of ofloxacin, the growth rate of $T$. denticola KT-3 was higher than that of the wildtype. These results indicated that inactivation of tepA2 might influence the susceptibility of $T$. denticola to these antimicrobials.

\section{DNA microarray analysis of the tepA2-deficient mutant}

In the tepA2-deficient mutant, susceptibility against kanamycin decreased while that to chloramphenicol and ofloxacin increased. To investigate the mechanisms of the increase in susceptibility against chloramphenicol, the gene expression profiles of T. denticola ATCC 35405 and KT-3 during exposure to chloramphenicol were compared using microarrays. Significantly differentially expressed genes in KT-3 relative to the wild-type strain are shown in Tables 3 and 4. Expression of the GNAT family acetyltransferase, glucosamine-6-phosphate deaminase, precorrin-4 C11-methyltransferase, several

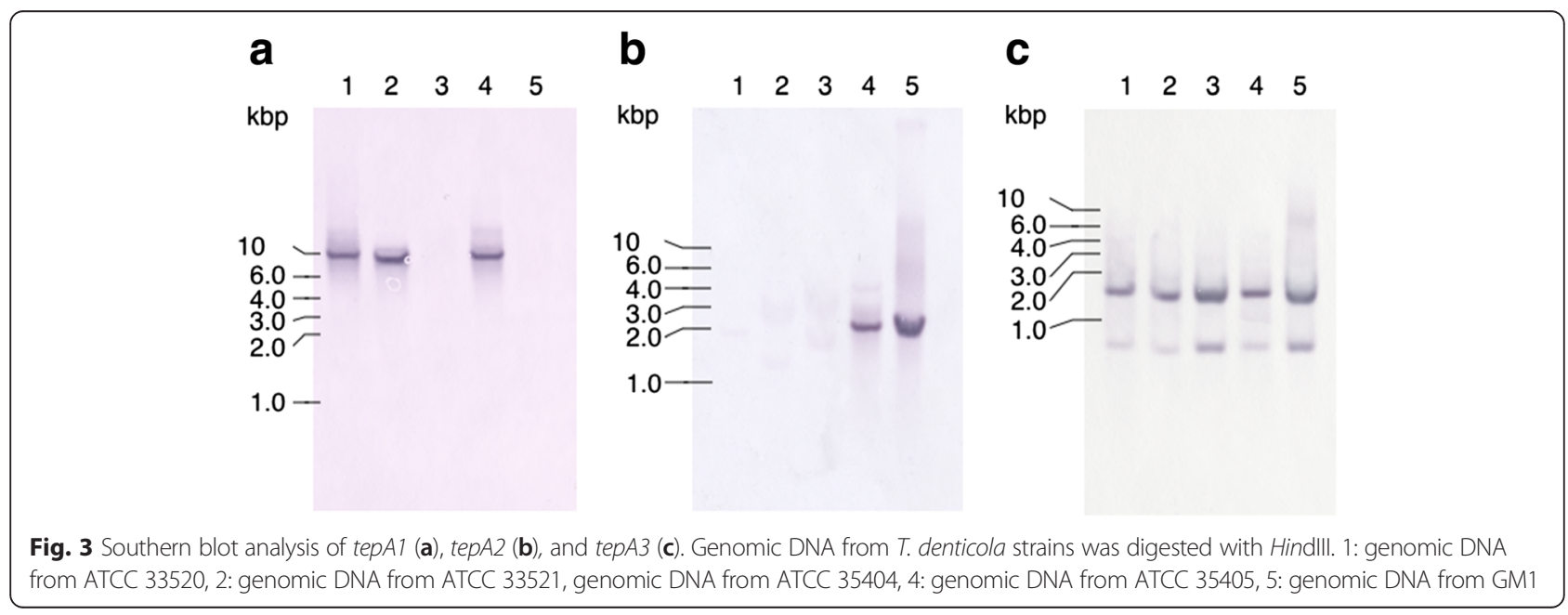




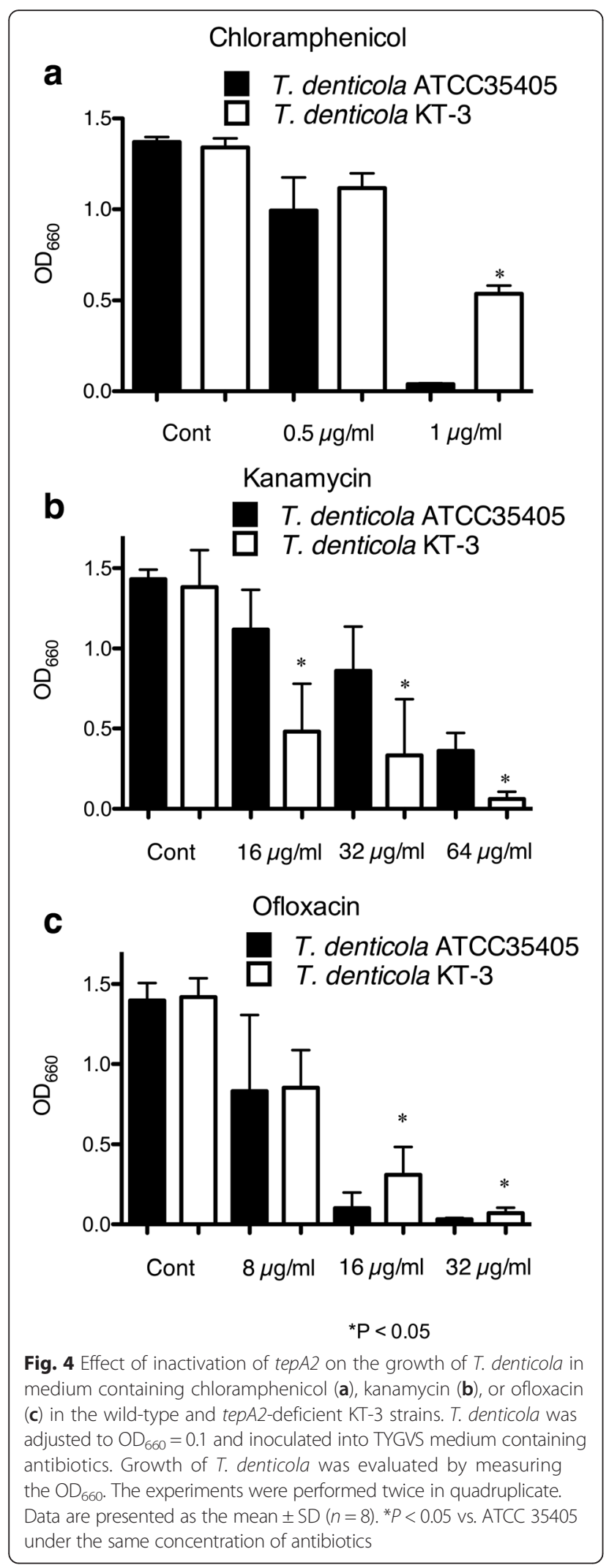

Table 3 Genes with increased expression in the tepA2-deficient mutant in the presence of chloramphenicol

\begin{tabular}{|c|c|}
\hline Gene & $\begin{array}{l}\text { Gene expression fold change } \\
\text { (KT-3 versus wild type) }\end{array}$ \\
\hline TDE_0499 hypothetical protein & 50.5 \\
\hline TDE_2748 acetyltransferase, GNAT family & 30.0 \\
\hline $\begin{array}{l}\text { TDE_0337 glucosamine-6-phosphate } \\
\text { deaminase }\end{array}$ & 19.5 \\
\hline TDE_2214 hypothetical protein & 17.9 \\
\hline TDE_0561 hypothetical protein & 16.0 \\
\hline $\begin{array}{l}\text { TDE_0614 precorrin-4 C11- } \\
\text { methyltransferase }\end{array}$ & 16.0 \\
\hline $\begin{array}{l}\text { TDE_0506 DNA-damage-inducible } \\
\text { protein J, putative }\end{array}$ & 15.3 \\
\hline TDE_1848 hypothetical protein & 15.2 \\
\hline TDE_0307 hypothetical protein & 14.3 \\
\hline $\begin{array}{l}\text { TDE_2378 ABC transporter, ATP-binding } \\
\text { protein, putative }\end{array}$ & 14.2 \\
\hline $\begin{array}{l}\text { TDE_0259 transcriptional regulator, } \\
\text { MarR family }\end{array}$ & 12.7 \\
\hline $\begin{array}{l}\text { TDE_1599 ABC transporter, ATP-binding/ } \\
\text { permease protein }\end{array}$ & 12.5 \\
\hline TDE_0551 hypothetical protein & 11.4 \\
\hline $\begin{array}{l}\text { TDE_0820 transcriptional regulator, } \\
\text { TetR family }\end{array}$ & 11.3 \\
\hline TDE_1517 hypothetical protein & 11.1 \\
\hline TDE_1692 hypothetical protein & 11.1 \\
\hline TDE_0528 hypothetical protein & 11.0 \\
\hline $\begin{array}{l}\text { TDE_0475 ABC transporter, ATP-binding } \\
\text { protein }\end{array}$ & 11.0 \\
\hline TDE_2519 hypothetical protein & 10.5 \\
\hline $\begin{array}{l}\text { TDE_0231 DNA polymerase III, } \\
\text { beta subunit }\end{array}$ & 10.3 \\
\hline TDE_0382 hypothetical protein & 9.8 \\
\hline TDE_2638 hypothetical protein & 9.5 \\
\hline $\begin{array}{l}\text { TDE_0375 ABC transporter, ATP-binding } \\
\text { protein }\end{array}$ & 9.4 \\
\hline TDE_1977 hypothetical protein & 9.3 \\
\hline $\begin{array}{l}\text { TDE_0748 iron compound ABC } \\
\text { transporter, periplasmic iron } \\
\text { compound-binding protein, putative }\end{array}$ & 8.8 \\
\hline $\begin{array}{l}\text { TDE_0426 bacteriocin ABC transporter, } \\
\text { ATP-binding/permease protein, putative }\end{array}$ & 6.1 \\
\hline $\begin{array}{l}\text { TDE_2431 bacteriocin ABC transporter, } \\
\text { ATP-binding/permease protein, putative }\end{array}$ & 2.8 \\
\hline
\end{tabular}

$\mathrm{ABC}$ transporters, and potential transcriptional regulators including TDE_0820 (transcriptional regulator, TetR family) was increased, while that of methyltransferase domain protein, several $A B C$ transporters, and GGDEF domain proteins, as well as pyrrolidonecarboxylate peptides were decreased. Increases in expression of tepA1 and tepA3 were detected in the 
Table 4 Genes with decreased expression in the tepA2-deficient mutant in the presence of chloramphenicol

\begin{tabular}{ll}
\hline & $\begin{array}{c}\text { Gene expression fold change } \\
\text { (KT-3 versus wild type) }\end{array}$ \\
\hline TDE_0719 bacteriocin ABC transporter, & 0.0004 \\
ATP-binding/permease protein, putative & \\
TDE_1057 hypothetical protein & 0.02 \\
TDE_1181 methyltransferase domain & 0.03 \\
protein & \\
TDE_2761 hypothetical protein & 0.05 \\
TDE_0953 branched-chain amino acid & 0.05 \\
ABC transporter, permease protein & \\
TDE_1883 hypothetical protein & 0.06 \\
TDE_1066 hypothetical protein & 0.06 \\
TDE_2582 GGDEF domain protein & 0.06 \\
TDE_1058 hypothetical protein & 0.06 \\
TDE_0720 bacteriocin ABC transporter, & 0.07 \\
bacteriocin-binding protein, putative & \\
TDE_0998 hypothetical protein & 0.09 \\
TDE_1930 hypothetical protein & 0.1 \\
TDE_0625 ABC transporter, ATP-binding & 0.1 \\
protein & 0.1 \\
TDE_0175 pyrrolidone-carboxylate & 0.1 \\
peptidase & 0.1 \\
TDE_1921 hypothetical protein & 0.1 \\
TDE_0485 hypothetical protein & 0.1 \\
TDE_0849 hypothetical protein & \\
TDE_1446 hypothetical protein & 0.1 \\
TDE_0894 hypothetical protein & \\
TDE_2497 hypothetical protein & 0.1 \\
TDE_0912 hypothetical protein & \\
TDE_0243 ABC transporter, ATP-binding & protein \\
TDE_2785 hypothetical protein & \\
TDE_1975 hypothetical protein & \\
TDE_0485 hypothetical protein & \\
\hline & \\
\hline
\end{tabular}

mutant; however, they were weak when compared to those of the listed genes.

\section{Expression of tepA1-B1, tepA3-B3, and TDE_0820 in the tepA2 mutant}

Among the genes that showed increased expression in the presence of chloramphenicol in KT-3 were TDE_0259 (transcriptional regulator, MarR family) and TDE_0820, which code for potential repressor proteins. We selected TDE_0820 for further evaluation by qRT-PCR because the change of expression between the wild type and the mutant was somewhat similar to that of TDE_0259 but its expression level in the wild type and the mutant was approximately 10 times higher than that of TDE_0259.
Increases in tep $A 1$ and tep $A 3$ expression were detected, although these were lower than those of the other genes in Table 3. To investigate the relationship among the bacteriocin $\mathrm{ABC}$ transporter genes, tepA1-B1, tepA3-B3 were also selected for further evaluation by qRT-PCR. The expression of tepA1 and tepB1 was not affected by the inactivation of tepA2 (Fig. 5a and b). The expression of tep $A 3$ and tepB3 was significantly increased by inactivation of tepA2 (Fig. 5c and d), indicating that it is associated with tepA2-B2 expression. In the presence of chloramphenicol, tep $A 1$ expression was decreased in both the wild-type strain and KT-3 (Fig. 5a). The expression of tepA3-B3 did not change with chloramphenicol treatment in the wild-type strain while it decreased in KT-3 (Fig. 5b and $\mathrm{c}$ ). The expression of TDE_0820 was increased in both the wild-type and KT-3 strains upon chloramphenicol treatment; however, the change was significant only in KT-3 (Fig. 5e). These results indicated that the increased expression of tepA3-B3 was induced by inactivation of tepA 2 and was independent of exposure to chloramphenicol. Expression of TDE_0820 in KT-3 suggested an association with chloramphenicol sensitivity.

\section{Discussion}

TepA2 shows 42-44 \% identity with the bacteriocin exporters of $S$. africana and C. lentocellum as well as with two orthologs, TepA1 and TepA3, of T. denticola ATCC 35405. The putative active-site residues for bacteriocin peptidases, membrane-spanning domains, and ATP-binding sites were conserved in all of the orthologs. Downstream of the orthologs, sequences for accessory proteins (TepBs), which showed similarity to the functional domain of hlydlike domains, were found. Although bacteriocin production has not been reported in $T$. denticola, putative proteins with double-glycine signal peptides, TDE_0416, TDE_0422, and TDE_0424 were located upstream of tepA1. These results tentatively suggest that tepA-tep $B$ may code for exporter proteins for these proteins.

The Southern blot analysis results indicated that the number of orthologs of tepA differs among $T$. denticola strains. Similarly, diversity among $T$. denticola strains has been reported for Msp (major sheath protein) [34]. However, diversity in the number of orthologs has not yet been documented. Msps are 53-63 kDa and the identity of the amino acid sequences between $T$. denticola strains ATCC 35405 and OKT is $43 \%$ [34]. The amino acid identity among tepAs (40\%) was similar to that observed among Msps. Only tepA3 was detected in all tested strains of $T$. denticola. The genes coding proteins with a bacteriocin-like leader peptide were detected only upstream of tep $A$. It is possible that tep $A$ and tep $B$, but not the bacteriocin-like genes, were duplicated during the evolution of $T$. denticola although further analysis is required to clarify the duplication events. 


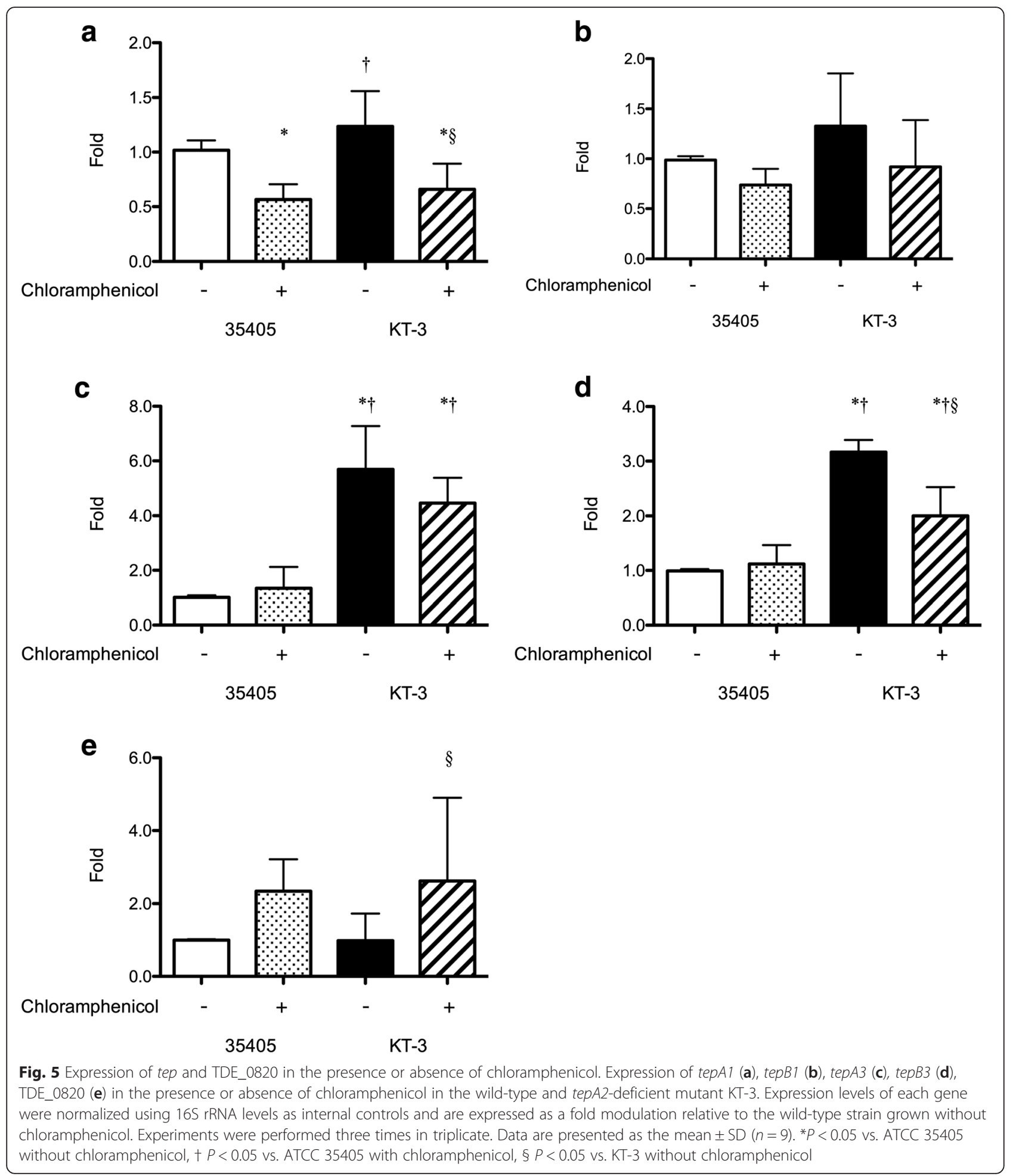

In the tepA2-deficient mutant, the expression of tepA3$B 3$ increased significantly while that of tepA1-B1 increased only slightly. In $T$. denticola, regulation of an $A B C$ transporter has been reported only for a thiamine pyrophosphate transporter, which is regulated by a TPP-binding riboswitch [35]. TepA2-B2 and tepA3-B3 have similarity to the functional motif for bacteriocin $\mathrm{ABC}$ transporters but they are not proximal to bacteriocin-like genes. Only tepA1-B1 has a bacteriocin-like protein directly upstream. A recent report indicated that peptides secreted through a bacteriocin export system could have signaling functions [36]. It is possible that a reduction in the export of a 
signaling molecule by tepA 2 may affect the expression of tepA3-B3. The expression of tepA3-B3 increased in the tepA2 mutant, while that of tepA1-B1 did not. These results suggest an interaction between regulation of expression of tepA2-B2 and that of tep $A 3-B 3$, and that regulation of tepA1-B1 is independent of tepA2-B2 and tepA3-B3.

Interestingly, resistance to chloramphenicol and ofloxacin was increased while resistance to kanamycin was reduced in the tep2-deficient mutant under conditions that induced increased tepA3 expression. Bacterial efflux pumps, including $\mathrm{ABC}$ transporters, are involved in drug resistance in several bacteria [37]. In $T$. denticola, resistance to antimicrobial agents such as human $\beta$-defensin 2 and 3, and rifampicin has been reported [38, 39], and an $A B C$ transporter was suggested to be involved in resistance to $\beta$-defensin 3 . In the microarray analysis, several genes encoding $A B C$ transporters and potential transcriptional regulators including TDE_0820 showed increased expression in the tepA2 mutant as compared to the wild-type strain. In addition, an acetyltransferase of the GNAT family exhibited significantly increased expression in the tepA2 mutant. These changes can potentially affect the susceptibility against chloramphenicol. However, the substrate of the enzyme group was reported to be kanamycin [40]; thus, the involvement of the enzyme in chloramphenicol resistance seems unlikely. The expression of tepA3-B3 in the KT-3 mutant was higher than that in the wild-type strain, although the level was low when compared to that in the mutant without chloramphenicol. It is possible that the changes in the expression of tepA3-B3 are required for sensitivity to chloramphenicol. Obviously, further analysis is required to define the role of these proteins with putative $A B C$ transporter functions in relation to sensitivity to the three antibiotics tested in this study.

\section{Conclusions}

T. denticola ATCC 35405 has three potential bacteriocin export proteins and the presence of these genes differs among the Treponema strains. Furthermore, TepA3-B3 of the proteins may be involved in resistance to chloramphenicol. The changes in susceptibility in T. denticola may contribute to our knowledge of the use of chemotherapy for chronic periodontitis.

\section{Abbreviations \\ ABC transporters, ATP-binding cassette transporters; ImmA, bacteriocin immuniry protein; NCBI, National Center for Biotechnology Information; Tep, Treponema exporter protein}

\section{Acknowledgements}

The authors thank Tomomi Kita for her technical assistance.

\section{Funding}

This work was partially supported by Grant 24592778 (K.I.) and 15 K1 1023 (K.I.) from the Ministry of Education, Science, Sport, Culture and Technology of Japan.

\section{Availability of data and materials}

The datasets except the array data supporting the conclusions of this article are included within the article. The array data in this report have been deposited in NCBI's Gene Expression Omnibus and are accessible through GEO series accession number GSE83445 (http://www.ncbi.nlm.nih.gov/geo/query/ acc.cgi?acc=GSE83445).

\section{Authors' contributions}

HKK, MY, and Kl designed the research. KT-K, YS-K, and HKK carried out the screening of bacteriocin-associated genes from $T$. denticola and in silico analysis of the genes. $K T-K, Y K$, and $Y S-K$ investigated the prevalence of bacteriocin-associated genes in the strains of $T$. denticola. KT-K, YK, and SS performed the gene expression analysis. KT-K, MY, and KI wrote the paper. All authors read and approved the final manuscript.

\section{Competing interests}

The authors declare that they have no competing interests.

\section{Consent to publish}

Not applicable.

\section{Ethics approval and consent to participate}

Not applicable.

\section{Author details}

'Department of Pediatric Dentistry, Tokyo Dental College, 2-9-18 Misaki-cho, Chiyoda-ku, Tokyo 101-0061, Japan. 'Department of Microbiology, Tokyo Dental College, 2-9-18 Misaki-cho, Chiyoda-ku, Tokyo 101-0061, Japan. ${ }^{3}$ Oral Health Science Center, Tokyo Dental College, 2-9-18 Misaki-cho, Chiyoda-ku, Tokyo 101-0061, Japan. ${ }^{4}$ Department of Oral Biology, State University of New York, Buffalo, NY, USA.

Received: 11 March 2016 Accepted: 2 July 2016

Published online: 16 July 2016

References

1. Socransky SS, Haffajee AD. Dental biofilms: difficult therapeutic targets. Periodontol 2000. 2002;28:12-55.

2. Papapanou PN, Baelum V, Luan WM, Madianos PN, Chen X, Fejerskov O, Dahlen G. Subgingival microbiota in adult Chinese: prevalence and relation to periodontal disease progression. J Periodontol. 1997;68:651-66.

3. Ishihara K. Virulence factors of Treponema denticola. Periodontol 2000. 2010; 54:117-35.

4. Socransky SS, Haffajee AD, Cugini MA, Smith C, Kent RL. Microbial complexes in subgingival plaque. J Clin Periodontol. 1998;25:134-44.

5. Teles RP, Haffajee AD, Socransky SS. Microbiological goals of periodontal therapy. Periodontol 2000. 2006;42:180-218.

6. Griffen AL, Beall CJ, Campbell JH, Firestone ND, Kumar PS, Yang ZK, Podar M, Leys EJ. Distinct and complex bacterial profiles in human periodontitis and health revealed by 165 pyrosequencing. ISME J. 2012;6:1176-85.

7. Jenkinson HF, Lamont RJ. Oral microbial communities in sickness and in health. Trends Microbiol. 2005;13:589-95.

8. Kolenbrander PE, Palmer Jr RJ, Rickard AH, Jakubovics NS, Chalmers NI, Diaz $\mathrm{PI}$. Bacterial interactions and successions during plaque development. Periodontol 2000. 2006:42:47-79.

9. Kuboniwa M, Tribble GD, James CE, Kilic AO, Tao L, Herzberg MC, Shizukuishi S, Lamont RJ. Streptococcus gordonii utilizes several distinct gene functions to recruit Porphyromonas gingivalis into a mixed community. Mol Microbiol. 2006; 60:121-39.

10. Testa MM, Ruiz de Valladares R, Benito de Cardenas IL. Antagonistic interactions among Fusobacterium nucleatum and Prevotella intermedia with oral lactobacilli. Res Microbiol. 2003;154:669-75.

11. Weerkamp A, Bongaerts-Larik L, Vogels GD. Bacteriocins as factors in the in vitro interaction between oral streptococci in plaque. Infect Immun. 1977; 16:773-80.

12. Kuramitsu HK, He X, Lux R, Anderson MH, Shi W. Interspecies interactions within oral microbial communities. Microbiol Mol Biol Rev. 2007;71:653-70.

13. Heng NC, Tagg JR, Tompkins GR. Competence-dependent bacteriocin production by Streptococcus gordonii DL1 (Challis). J Bacteriol. 2007;189:1468-72. 
14. Apolonio AC, Carvalho MA, Bemquerer MP, Santoro MM, Pinto SQ, Oliveira JS Santos KV, Farias LM. Purification and partial characterization of a bacteriocin produced by Eikenella corrodens. J Appl Microbiol. 2008;104:508-14.

15. Nakamura T, Fujimura S, Obata N, Yamazaki N. Bacteriocin-like substance (melaninocin) from oral Bacteroides melaninogenicus. Infect Immun. 1981;31: 28-32.

16. Hammond BF, Lillard SE, Stevens RH. A bacteriocin of Actinobacillus actinomycetemcomitans. Infect Immun. 1987;55:686-91.

17. Takazoe I, Nakamura T, Okuda K. Colonization of the subgingival area by Bacteroides gingivalis. J Dent Res. 1984;63:422-6.

18. Kamiya RU, Napimoga MH, Hofling JF, Goncalves RB. Frequency of four different mutacin genes in Streptococcus mutans genotypes isolated from caries-free and caries-active individuals. J Med Microbiol. 2005:54:599-604.

19. Baba T, Schneewind O. Instruments of microbial warfare: bacteriocin synthesis, toxicity and immunity. Trends Microbiol. 1998;6:66-71.

20. Bastos Mdo C, Coelho ML, Santos OC. Resistance to bacteriocins produced by Gram-positive bacteria. Microbiology. 2015;161:683-700.

21. Dirix G, Monsieurs P, Dombrecht B, Daniels R, Marchal K, Vanderleyden J, Michiels J.Peptide signal molecules and bacteriocins in Gram-negative bacteria: a genome-wide in silico screening for peptides containing a double-glycine leader sequence and their cognate transporters. Peptides. 2004;25:1425-40.

22. Grenier D. Nutritional interactions between two suspected periodontopathogens, Treponema denticola and Porphyromonas gingivalis. Infect Immun. 1992;60: 5298-52301.

23. Grenier D. Antagonistic effect of oral bacteria towards Treponema denticola. J Clin Microbiol. 1996;34:1249-52.

24. Hyink O, Wescombe PA, Upton M, Ragland N, Burton JP, Tagg JR. Salivaricin $\mathrm{A} 2$ and the novel lantibiotic salivaricin B are encoded at adjacent loci on a 190-kilobase transmissible megaplasmid in the oral probiotic strain Streptococcus salivarius K12. Appl Environ Microbiol. 2007:73:1107-13.

25. Novak J, Caufield PW, Miller EJ. Isolation and biochemical characterization of a novel lantibiotic mutacin from Streptococcus mutans. J Bacteriol. 1994;176:4316-20

26. Yonezawa H, Kuramitsu HK. Genetic analysis of a unique bacteriocin, Smb, produced by Streptococcus mutans GS5. Antimicrob Agents Chemother. 2005:49:541-8.

27. Ohta K, Mäkinen KK, Loesche WJ. Purification and characterization of an enzyme produced by Treponema denticola capable of hydrolyzing synthetic trypsin substrates. Infect Immun. 1986;53:213-20.

28. Matsumoto-Nakano M, Kuramitsu HK. Role of bacteriocin immunity proteins in the antimicrobial sensitivity of Streptococcus mutans. J Bacteriol. 2006;188: 8095-102.

29. Ishihara K, Kuramitsu HK, Miura T, Okuda K. Dentilisin activity affects the organization of the outer sheath of Treponema denticola. J Bacteriol. 1998; 180:3837-44.

30. Horton RM, Ho SN, Pullen JK, Hunt HD, Cai Z, Pease LR. Gene splicing by overlap extension. Methods Enzymol. 1993;217:270-9.

31. Fath MJ, Kolter R. ABC transporters: bacterial exporters. Microbiol Rev. 1993; 57:995-1017.

32. Wu KH, Tai PC. Cys32 and His105 are the critical residues for the calciumdependent cysteine proteolytic activity of CvaB, an ATP-binding cassette transporter. J Biol Chem. 2004;279:901-9.

33. Gaudet R, Wiley DC. Structure of the ABC ATPase domain of human TAP1, the transporter associated with antigen processing. EMBO J. 2001;20:4964-72.

34. Fenno JC, Wong GW, Hannam PM, Muller KH, Leung WK, McBride BC. Conservation of $m s p$, the gene encoding the major outer membrane protein of oral Treponema spp. J Bacteriol. 1997;179:1082-9.

35. Bian J, Shen H, Tu Y, Yu A, Li C. The Riboswitch Regulates a Thiamine Pyrophosphate ABC Transporter of the Oral Spirochete Treponema denticola. J Bacteriol. 2011;193:3912-22.

36. Michiels J, Dirix G, Vanderleyden J, Xi C. Processing and export of peptide pheromones and bacteriocins in Gram-negative bacteria. Trends Microbiol. 2001;9:164-8.

37. Sun J, Deng Z, Yan A. Bacterial multidrug efflux pumps: mechanisms, physiology and pharmacological exploitations. Biochem Biophys Res Commun. 2014; 453:254-67.

38. Brissette CA, Lukehart SA. Treponema denticola is resistant to human betadefensins. Infect Immun. 2002;70:3982-4.

39. Cheng SL, Siboo R, Quee TC, Johnson JL, Mayberry WR, Chan EC. Comparative study of six random oral spirochete isolates. Serological heterogeneity of Treponema denticola. J Periodontal Res. 1985;20:602-12.
40. Vetting MW, SdC LP, Yu M, Hegde SS, Magnet S, Roderick SL, Blanchard JS Structure and functions of the GNAT superfamily of acetyltransferases. Arch Biochem Biophys. 2005:433:212-26.

41. Chan EC, Siboo R, Keng T, Psarra N, Hurley R, Cheng SL, lugovaz I. Treponema denticola (ex Brumpt 1925) sp. nov., nom. rev., and identification of new spirochete isolates from periodontal pockets. Int J Syst Bacteriol. 1993; 43:196-203.

42. Weinberg A, Holt SC. Interaction of Treponema denticola TD-4, GM-1, and MS25 with human gingival fibroblasts. Infect Immun. 1990;58:1720-9.

\section{Submit your next manuscript to BioMed Central and we will help you at every step:}

- We accept pre-submission inquiries

- Our selector tool helps you to find the most relevant journal

- We provide round the clock customer support

- Convenient online submission

- Thorough peer review

- Inclusion in PubMed and all major indexing services

- Maximum visibility for your research

Submit your manuscript at www.biomedcentral.com/submit
Biomed Central 